\title{
Higgs boson decay into a lepton pair and a photon revisited
}

\author{
Aliaksei Kachanovich $\odot,{ }^{*}$ Ulrich Nierste $\odot,{ }^{\dagger}$ and Ivan Nišandžic ${ }^{\star}$ \\ Institut für Theoretische Teilchenphysik (TTP), Karlsruher Institut für Technologie (KIT), \\ 76131 Karlsruhe, Germany
}

(Received 29 January 2020; accepted 4 March 2020; published 16 April 2020; corrected 16 December 2020)

\begin{abstract}
We present new calculations of the differential decay rates for $H \rightarrow \ell^{+} \ell^{-} \gamma$ with $\ell=e$ or $\mu$ in the Standard Model. The branching fractions and forward-backward asymmetries, defined in terms of the flight direction of the photon relative to the lepton momenta, depend on the cuts on energies and invariant masses of the final state particles. For typical choices of these cuts we find the branching ratios $B(H \rightarrow e \bar{e} \gamma)=$ $5.8 \times 10^{-5}$ and $B(H \rightarrow \mu \bar{\mu} \gamma)=6.4 \times 10^{-5}$ and the forward-backward asymmetries $\mathcal{A}_{\mathrm{FB}}^{(e)}=0.343$ and $\mathcal{A}_{\mathrm{FB}}^{(\mu)}=0.255$. We provide compact analytic expressions for the differential decay rates for the use in experimental analyses.
\end{abstract}

DOI: 10.1103/PhysRevD.101.073003

\section{INTRODUCTION}

Since the discovery of a Higgs boson with a mass of $125 \mathrm{GeV}[1,2]$ in 2012, the LHC experiments CMS and ATLAS put a major effort into the precise determination of its couplings. The Standard Model (SM) accommodates a minimal Higgs sector, with just one Higgs doublet, and it is natural to ask whether nature foresees a richer Higgs sector than the SM. A possible imprint of an extended Higgs sector are deviations of the measured couplings from their SM predictions [3]. To date CMS and ATLAS have studied the couplings of the discovered Higgs boson to $W$ [4-7] and $Z$ [7-10] bosons, $\tau$ leptons [11-13], $b[14,15]$ and $t[16,17]$ quarks, as well as photons $[18,19]$. The latter coupling is loop suppressed and has been probed though the rare decay $H \rightarrow \gamma \gamma$. Rare Higgs decays are especially sensitive to physics beyond the SM and even probe scenarios with only one Higgs doublet as in the SM. For instance, $H \rightarrow \gamma \gamma$ data were instrumental to rule out a fourth sequential fermion generation from a global analysis of Higgs signal strengths [20]. Phenomenological analyses of two-Higgs-doublet models usually assume simple versions of the Yukawa sector (called type I, II, X, or Y), in which different observables become correlated and the largest imprints are on the heavy fermions of the third generation [21-31]. In such models the dynamics of light fermions of

\footnotetext{
*aliaksei.kachanovich@kit.edu

†ulrich.nierste@kit.edu

ivan.nisandzic@kit.edu
}

Published by the American Physical Society under the terms of the Creative Commons Attribution 4.0 International license. Further distribution of this work must maintain attribution to the author(s) and the published article's title, journal citation, and DOI. Funded by SCOAP. the first and second generation follow the pattern of the third generation and measurements of Higgs decay rates involving light fermions will only provide redundant information. However, as outlined in the following paragraph there are well-motivated phenomenological reasons to consider the possibility that physics beyond the SM shows imprints on the decays of the $125 \mathrm{GeV}$ Higgs bosons into final states containing light fermions. It is therefore mandatory to measure the corresponding decay rates accurately and to compare the data with precise SM predictions.

In this paper we study the SM predictions for the rare decays $H \rightarrow \ell^{+} \ell^{-} \gamma$ with focus on $\ell=e$ and $\ell=\mu$. While the amplitude of $H \rightarrow \ell^{+} \ell^{-}$is suppressed by one power of the Yukawa coupling $y_{\ell}=m_{\ell} / v$, where $m_{\ell}$ is the lepton mass and $v=174 \mathrm{GeV}$ is the vacuum expectation value of the Higgs field, the radiative analogue does not suffer from this suppression.

Electroweak loop contributions instead involve the Higgs coupling to heavy gauge bosons or to the top quark and permit a nonzero decay amplitude even for $y_{\ell}=0$, producing the lepton pair in a state with angular momentum $j=1$. The decay rate $\Gamma\left(H \rightarrow e^{+} e^{-} \gamma\right)$ exceeds $\Gamma\left(H \rightarrow e^{+} e^{-}\right)$by far, while $\Gamma\left(H \rightarrow \mu^{+} \mu^{-} \gamma\right)$ and $\Gamma\left(H \rightarrow \mu^{+} \mu^{-}\right)$are comparable in size. In the case of $\Gamma\left(H \rightarrow \tau^{+} \tau^{-} \gamma\right)$ the electroweak loop contribution is much smaller than the tree-level contribution proportional to $y_{\tau}^{2}$, which simply amounts to the bremsstrahlung contribution to $H \rightarrow \tau^{+} \tau^{-}$. It is important to note that $H \rightarrow \ell^{+} \ell^{-}$and $H \rightarrow \ell^{+} \ell^{-} \gamma$ probe different sectors of beyond the Standard Model (BSM) models (chirality-flipping vs chirality-conserving couplings to lepton fields) and are therefore complementary. A further motivation to study $H \rightarrow \mu^{+} \mu^{-} \gamma$ is the $3.7 \sigma$ discrepancy between the measured anomalous magnetic moment of the muon, $a_{\mu}$, and its SM prediction [32]. $a_{\mu}$ involves the magnetic operator $\bar{L}_{\mu} \Phi \sigma_{\alpha \beta} \mu_{R} F^{\alpha \beta}$, with the lepton doublet 
$L_{\mu}=\left(\nu_{\mu}, \mu\right)$, the Higgs doublet $\Phi$, and the electromagnetic field strength tensor $F^{\alpha \beta}$. BSM models with loop contributions to the coefficient of the magnetic operator may as well affect the $H-\bar{\mu}-\mu-\gamma$ couplings. Another related topic are the hints of violation of lepton flavor universality encoded in the ratios $R_{K^{(*)}} \equiv B\left(B \rightarrow K^{(*)} \mu^{+} \mu^{-}\right) / B\left(B \rightarrow K^{(*)} e^{+} e^{-}\right)$ $[33,34]$, which support BSM physics coupling to left-chiral leptons $[35,36]$. Also here the underlying BSM dynamics can eventually be tested with $H \rightarrow \ell^{+} \ell^{-} \gamma$. None of the $H \rightarrow \ell^{+} \ell^{-} \gamma$ decays has been observed yet; cf. Ref. [37] for LHC limits.

Analytic expressions for differential $H \rightarrow \ell^{+} \ell^{-} \gamma$ decay rates have been derived in Refs. [38,39]. After the discovery of the $125 \mathrm{GeV}$ Higgs boson Ref. [38] was updated [40] and two new detailed analyses based on novel calculations of the decay rate have been presented in Refs. [41,42]. Comparing the decay rates $d \Gamma\left(H \rightarrow \ell^{+} \ell^{-} \gamma\right) / d \sqrt{s}$, where $\sqrt{s}$ is the invariant mass of the lepton pair, presented in these papers, we find significant discrepancies, which motivates the new calculation of this decay rate presented in this paper. We use a linear $R_{\xi}$ gauge, so that we can use the vanishing of the $W$ and $Z$ gauge parameters as a check of our calculation. This check is especially valuable in the context of the $Z$ width, which must be taken into account when the invariant mass of the lepton pair is close to the $Z$ boson mass and special care is needed to ensure a gauge-independent result $[43,44]$. In this kinematic region our decay is indistinguishable from $H \rightarrow$ $Z \gamma$ (for recent LHC search limits cf. [45,46]). In Ref. [41] it is argued that $H \rightarrow Z \gamma$ is not a properly defined physical process and one should instead discuss the full decay chain, including the $Z$ decay, such as $H \rightarrow \ell^{+} \ell^{-} \gamma$. Since we keep the gauge parameters arbitrary, we can track how the unphysical gauge-dependent pieces of the $H \rightarrow Z\left[\rightarrow \ell^{+} \ell^{-}\right] \gamma$ subprocess cancel with those of other diagrams. We will quote a compact formula for the differential decay rate $d^{2} \Gamma(H \rightarrow$ $\left.\ell^{+} \ell^{-} \gamma\right) /(d s d t)$ with respect to the Mandelstam variables $s$ and $t$, where $\sqrt{t}$ is the invariant mass of the $\left(\ell^{-}, \gamma\right)$ pair, and discuss the forward-backward asymmetry of the photon.

Our paper is organized as follows: In the following section we present our calculation and discuss our results, including a comparison with the literature. Section III contains our conclusions, followed by two Appendixes guiding through our analytic results.

\section{CALCULATION AND RESULTS}

\section{A. Amplitudes}

The amplitude for the tree-level photon emission process (see Fig. 1) is

$$
\begin{aligned}
\mathcal{A}_{\text {tree }}= & -\frac{e^{2} m_{\ell} \varepsilon_{\nu}^{*}(k)}{2 m_{W} \sin \theta_{W}}\left[\frac{\bar{u}\left(p_{1}\right)\left(\gamma^{\nu} \not k+2 p_{1}^{\nu}\right) v\left(p_{2}\right)}{t-m_{\ell}^{2}}\right. \\
& \left.-\frac{\bar{u}\left(p_{1}\right)\left(\not k \gamma^{\nu}+2 p_{2}^{\nu}\right) v\left(p_{2}\right)}{u-m_{\ell}^{2}}\right],
\end{aligned}
$$

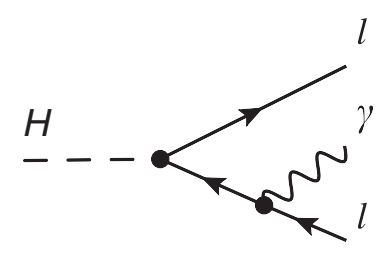

(a)

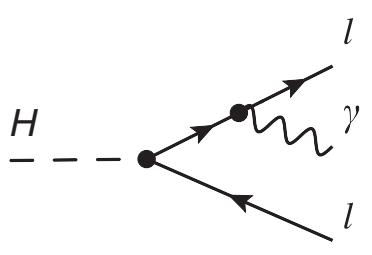

(b)
FIG. 1. Tree-level Feynman diagrams.

with our conventions for the kinematical variables as following: We denote four-momenta of the photon, lepton and antilepton by $k, p_{1}$, and $p_{2}$, respectively. Squared invariant masses are denoted by the Mandelstam variables $s=\left(p_{1}+p_{2}\right)^{2}, t=\left(p_{1}+k\right)^{2}$, and $u=\left(p_{2}+k\right)^{2}$ obeying the relation $s+t+u=m_{H}^{2}+2 m_{\ell}^{2}$, where $m_{H}$ is the Higgs boson mass. $e, m_{W}$, and $\theta_{W}$ are the electromagnetic coupling constant, mass of the $W$ boson, and weak mixing angle, respectively. $u$ and $v$ are the lepton and antilepton spinors and $\varepsilon$ is the polarization vector of the photon.

In the one-loop contribution we can neglect $y_{\ell}$. The Feynman diagrams may be grouped into several classes as depicted in Fig. 2. Sample diagrams can be found in Fig. 3. The one-loop amplitude can be parametrized as

$$
\begin{aligned}
\mathcal{A}_{\text {loop }}= & {\left[\left(k_{\mu} p_{1 \nu}-g_{\mu \nu} k \cdot p_{1}\right) \bar{u}\left(p_{2}\right)\left(a_{1} \gamma^{\mu} P_{R}+b_{1} \gamma^{\mu} P_{L}\right) v\left(p_{1}\right)\right.} \\
& +\left(k_{\mu} p_{2 \nu}-g_{\mu \nu} k \cdot p_{2}\right) \bar{u}\left(p_{2}\right)\left(a_{2} \gamma^{\mu} P_{R}+b_{2} \gamma^{\mu} P_{L}\right) \\
& \left.\times v\left(p_{1}\right)\right] \varepsilon^{\nu *}(k) .
\end{aligned}
$$

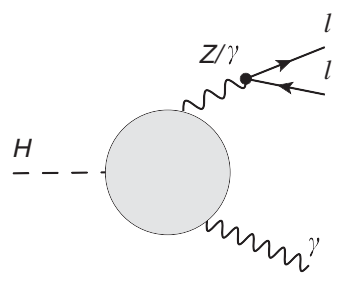

(a)

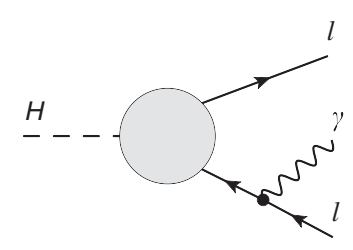

(c)

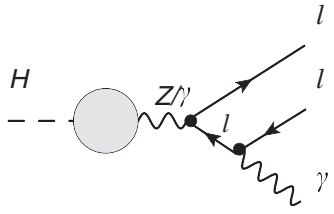

(b)

(d)

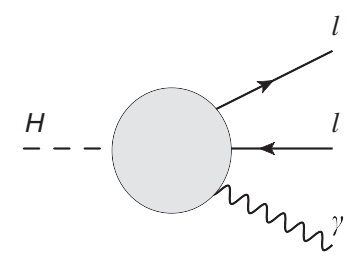

FIG. 2. Schematic representation of the classes of one-loop diagrams that contribute to $H \rightarrow \ell \ell \gamma$ at the one-loop level, with the blob representing one-particle irreducible diagrams. Total contribution from class (b) is vanishing. None of the classes shown in (a), (c), and (d) is separately gauge independent within linear $R_{\xi}$ gauge. 


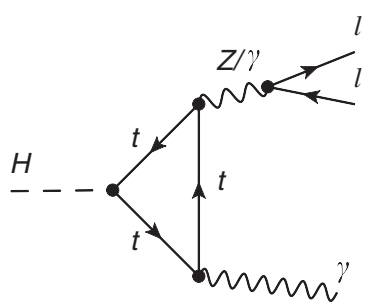

(a)

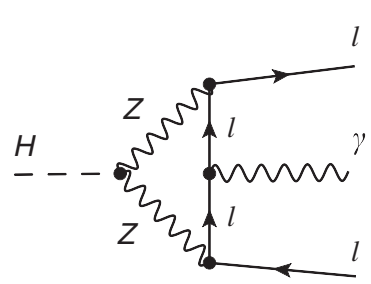

(d)

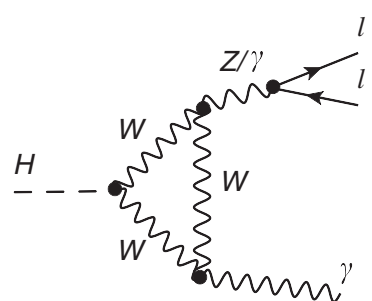

(b)

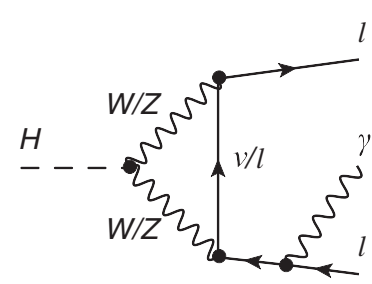

(e)

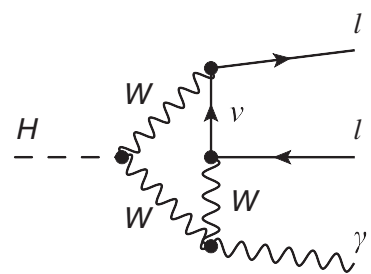

(c)

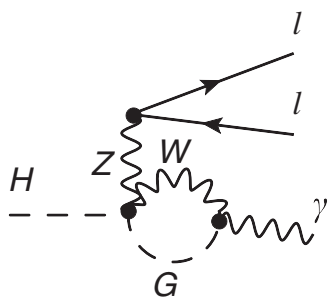

(f)

FIG. 3. Sample Feynman diagrams contributing to $H \rightarrow \ell \ell \gamma$ at one-loop level.

$P_{L, R}=\left(1 \mp \gamma_{5}\right) / 2$ are chiral projectors and the coefficients $a_{1,2}$ and $b_{1,2}$ are functions of $s, t, u$ and the particle masses. We present the analytic results for $a_{1}$ and $b_{1}$ in Appendix A in Eqs. (A1) and (A2); $a_{2}$ and $b_{2}$ are obtained by interchanging $t$ and $u: a_{2}(t, u)=a_{1}(u, t)$ and $b_{2}(t, u)=$ $b_{1}(u, t)$. The compact results in Eqs. (A1) and (A2) involve the coefficient functions appearing in the PassarinoVeltman decomposition $[47,48]$ of the tensor integrals. A result fully reduced to scalar one-loop functions [49,50] is given in the ancillary file [51] attached to this paper in terms of a Mathematica file.

We keep only the top quark in the fermion triangle diagrams while neglecting all other Yukawa couplings. This leaves us with 119 one-loop diagrams. We have explicitly checked that the final result is finite in the soft and collinear limits. This infrared (IR) safety must hold, because for massless leptons the one-loop result constitutes the leading contribution and there are no diagrams with a virtual photon to cancel any IR divergences.

We now comment on several differences with respect to existing results in the literature. References [39,52] contain additional terms of the form $\epsilon_{\mu \nu \rho \sigma} k^{\rho}\left(p_{1}+p_{2}\right)^{\sigma} \varepsilon^{\nu *}$ involving the Levi-Civita tensor in the final result for the loop amplitude. This contribution is absent in our result in Eq. (2). ${ }^{1}$ We have found that this term indeed appears in the top-quark triangle diagram (a) of Fig. 3 but cancels with the corresponding diagram with opposite fermion number flow. We have used the 't Hooft-Veltman scheme for the treatment of $\gamma_{5}$ in $D$ dimensions.

Using a nonlinear gauge [53] the authors of Ref. [38] have identified classes of diagrams which separately satisfy the electromagnetic Ward identity. Using instead the usual

\footnotetext{
${ }^{1}$ These terms are also absent in the final results of Refs. [38,53].
}

linear $R_{\xi}$ gauge we find a straightforward cancellation of the $Z$ boson gauge parameter $\xi_{Z}$, while the cancellation of the dependence on the $W$ boson gauge parameter $\xi_{W}$ involves subtleties: The tree-level relation $e^{2} / g_{2}^{2}=$ $\sin ^{2} \theta_{W}=1-m_{W}^{2} / m_{Z}^{2}$ (which is promoted to an all-order relation in the on-shell renormalization scheme for $\sin \theta_{W}$ ) is instrumental for the cancellation of $\xi_{W}$ from the result. But in order to describe the decay distribution for $\sqrt{s} \equiv$ $m_{\ell \ell}$ in the region around the $Z$ resonance, we must use a Breit-Wigner shape for the $Z$ propagator in the diagrams of class (a) in Fig. 2. Yet this modification spoils the cancellation of $\xi_{W}$ dependence between triangle and other diagrams; a remedy is the use of the complex-mass scheme introduced in [43,44] as e.g., done in Ref. [41]. We instead start with strictly real gauge boson masses, verify the $\xi_{W}$ independence of the result, and subsequently add the finite $Z$ width $\Gamma_{Z}$ to the final, gauge-independent result.

In most phase-space regions our loop functions are real; exceptions are kinematical situations such as $t>M_{W}^{2}$ or $u>M_{W}^{2}$ permitting on-shell cuts of the loops. Switching to the complex mass scheme makes the real loop expressions develop imaginary parts proportional to the gauge boson width; in the corresponding phase-space regions the decay rates found in the two approaches differ by terms quadratic in $\Gamma_{W, Z}$. We checked that the difference in $d \Gamma\left(H \rightarrow \ell^{+} \ell^{-} \gamma\right) / d \sqrt{s}$ between the two approaches is numerically negligible. Therefore the treatment of $\Gamma_{Z}$ cannot be the reason for the numerical differences between our result and the various results in the literature.

We next shortly describe the tools used in our calculation. We have generated the Feynman diagrams with the FeynArts package [54]. For the evaluation of the loop integrals and the reduction to scalar basis functions we have used FeynCalc [55,56] and have verified the 
cancellation of infrared poles in the final result using Package-X [57] linked to FeynCalc via the FeynHelpers package [58]. Then we have evaluated the loop functions using CollierLink [57,59] that provides a Mathematica link to the Collier package [60-62]. The LoopTools package [63,64] is finally used for additional numerical checks. For the numerical integration over the phase-space variables we use vegas [65] from the Cuba library [66] and Mathematica [67].

\section{B. Decay rates}

The tree contribution to the decay rate shown in Fig. 1 reads

$$
\begin{aligned}
\frac{d^{2} \Gamma_{\text {tree }}}{d s d t}= & \mathcal{N}\left[\frac{9 m_{\ell}^{4}+m_{\ell}^{2}(-2 s+t-3 u)+t u}{\left(t-m_{\ell}^{2}\right)^{2}}\right. \\
& +\frac{9 m_{\ell}^{4}+m_{\ell}^{2}(-2 s+u-3 t)+t u}{\left(u-m_{\ell}^{2}\right)^{2}} \\
& \left.+\frac{34 m_{\ell}^{4}-2 m_{\ell}^{2}(8 s+5(t+u))+2(s+t)(s+u)}{\left(t-m_{\ell}^{2}\right)\left(u-m_{\ell}^{2}\right)}\right],
\end{aligned}
$$

with

$$
\mathcal{N}=\frac{e^{4} m_{\ell}^{2}}{256 \pi^{3} \sin ^{2} \theta_{W} m_{W}^{2} m_{H}^{3}} .
$$

The contribution from the one-loop diagrams is

$\frac{d^{2} \Gamma_{\text {loop }}}{d s d t}=\frac{s}{512 \pi^{3} m_{H}^{3}}\left[t^{2}\left(\left|a_{1}\right|^{2}+\left|b_{1}\right|^{2}\right)+u^{2}\left(\left|a_{2}\right|^{2}+\left|b_{2}\right|^{2}\right)\right]$.

While we set $m_{\ell}$ to zero in Eq. (5), we retain a nonvanishing value of $m_{\ell}$ in the kinematical limits of the phase-space integration. The limits for the variables $s$ and $t$ can be expressed as

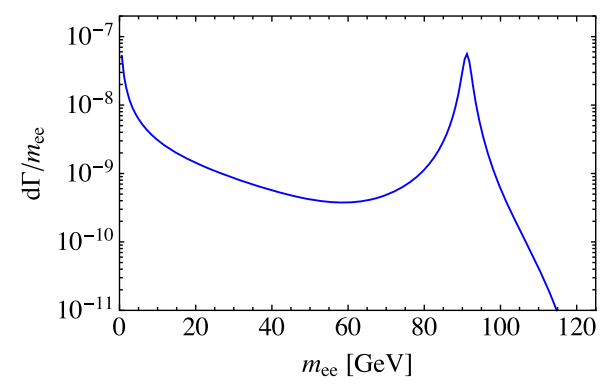

(a)

$$
\begin{aligned}
s_{\min } & =4 m_{\ell}^{2}, \quad s_{\max }=m_{H}^{2}, \\
t_{\min (\max )} & =\frac{1}{2}\left(m_{H}^{2}-s+2 m_{\ell}^{2} \mp\left(m_{H}^{2}-s\right) \sqrt{1-4 m_{\ell}^{2} / s}\right) .
\end{aligned}
$$

The tree-level contribution exhibits an infrared pole as $s$ approaches its maximum value $s_{\max }=m_{H}^{2}$ that corresponds to a vanishing photon energy $E_{\gamma}$ in the Higgs boson rest frame, $E_{\gamma}=\left(m_{H}^{2}-s\right) / 2 m_{H}$. For the evaluation of the total decay rate we impose the cut $E_{\gamma, \min }$ which lowers the maximum value of $s$ to $s_{\text {cut }}=m_{H}^{2}-2 m_{H} E_{\gamma, \min }$. The resulting differential decay rate over $m_{\ell \ell}=\sqrt{s}$, for $\ell=e, \mu$, is shown in Fig. 4. One notes the enhancement from the $Z$ pole as well as the tail of photon pole starting at $m_{\ell \ell \text {, } \min }$. The contribution of the interference between treelevel and one-loop contributions is negligible, as well as the effect of the tree-level diagrams in the case of electrons. For the evaluation of the full decay rates we employ the kinematical cuts of Refs. [40,41], namely:

$$
\begin{aligned}
s, t, u & >\left(0.1 m_{H}\right)^{2}, \quad E_{\gamma}>5 \mathrm{GeV}, \\
\left(E_{1}\right. & \left.>7 \mathrm{GeV}, \quad E_{2}>25 \mathrm{GeV}\right) \quad \text { or } \\
\left(E_{1}\right. & \left.>25 \mathrm{GeV}, \quad E_{2}>7 \mathrm{GeV}\right) .
\end{aligned}
$$

We use the following input for the physical parameters:

$$
\begin{aligned}
m_{W} & =80.379 \mathrm{GeV}, \quad m_{Z}=91.1876 \mathrm{GeV}, \\
\sin ^{2} \theta_{W} & =1-\frac{m_{W}^{2}}{m_{Z}^{2}}=0.223013, \\
m_{t} & =173.1 \mathrm{GeV}, \quad m_{H}=125.1 \mathrm{GeV}, \\
G_{F} & =1.1663787 \times 10^{-5} \mathrm{GeV}^{-2}, \\
\alpha^{-1} & =\frac{\pi}{\sqrt{2} G_{F} m_{W}^{2} \sin ^{2} \theta_{W}}=132.184 .
\end{aligned}
$$

Here $m_{t}$ is the top mass, $G_{F}$ is the Fermi constant, $\alpha=$ $e^{2} /(4 \pi)$ is the fine structure constant and the other quantities are defined after Eq. (1). Our calculation of $\alpha$

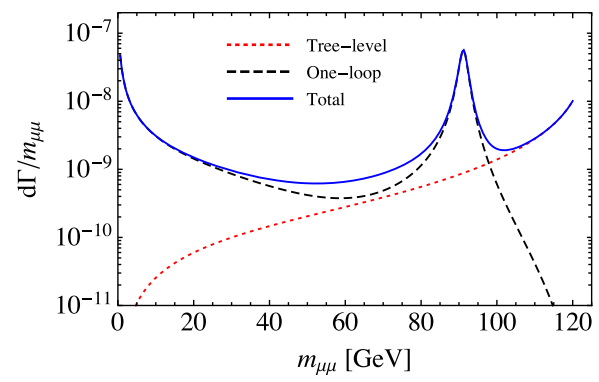

(b)

FIG. 4. Differential decay rate with respect to the invariant dilepton mass for (a) electrons and (b) muons. The tree-level, one-loop, and total contributions are denoted by red dotted, black dashed lines, and solid blue lines, respectively. The tree-level contribution for the case of electrons is negligible. The only cut which we impose for these plots is $E_{\gamma, \min }=5 \mathrm{GeV}$ which merely amounts to a lowering of the maximum value of $m_{\ell \ell}$. 
in Eq. (8) employs the tree-level relations between $G_{F}$ and the fundamental parameters of the SM, as e.g., in Ref. [68]. Radiative corrections shift this value to the familiar $\alpha^{-1} \simeq 128$, but the ambiguity stemming from the choices of numerical values for $\alpha$ and other inputs can only be resolved by performing a next-to-leading-order (e.g., twoloop) calculation of the $H \rightarrow \ell^{+} \ell^{-} \gamma$ decay rate.

The numerical values of the rates are

$$
\Gamma^{(e)}=0.237 \mathrm{keV}, \quad \Gamma^{(\mu)}=0.262 \mathrm{keV} .
$$

The difference between $\Gamma^{(e)}$ and $\Gamma^{(\mu)}$ stems from the treelevel contribution. Figure 5 shows the differential decay rate with respect to the invariant mass of the lepton-photon pair. With a total Higgs width of $4.1 \mathrm{MeV}$ the rates in Eq. (9) correspond to the branching ratios

$$
\begin{aligned}
& B(H \rightarrow e \bar{e} \gamma)=5.8 \times 10^{-5}, \\
& B(H \rightarrow \mu \bar{\mu} \gamma)=6.4 \times 10^{-5} .
\end{aligned}
$$

These branching ratios are roughly 3 times smaller than $B(H \rightarrow \mu \bar{\mu})$.

\section{Forward-backward asymmetry}

Here we present the differential decay distribution with respect to $\cos \theta^{(\ell)}$, where $\theta^{(\ell)}$ is the angle between the lepton and the photon in the rest frame of the Higgs boson, $t=E_{\gamma}\left(E_{1}-\left|\vec{p}_{1}\right| \cos \theta^{(\ell)}\right)$. The resulting distribution for the case of $\ell=\mu$ is shown in Fig. 6. For this evaluation we apply the cuts $m_{\mu \mu}>0.1 m_{H}$ and $E_{\gamma}>5 \mathrm{GeV}$ (and no cuts on $\left.E_{1,2}\right)$. We define the forward-backward asymmetry with respect to $\theta^{(\ell)}$ as

$$
\mathcal{A}_{\mathrm{FB}}^{(\ell)}=\frac{\int_{-1}^{0} \frac{d \Gamma}{d \cos \theta^{(\ell)}}-\int_{0}^{1} \frac{d \Gamma}{d \cos \theta^{(\ell)}}}{\int_{-1}^{0} \frac{d \Gamma}{d \cos \theta^{(\ell)}}+\int_{0}^{1} \frac{d \Gamma}{d \cos \theta^{(\ell)}}} .
$$

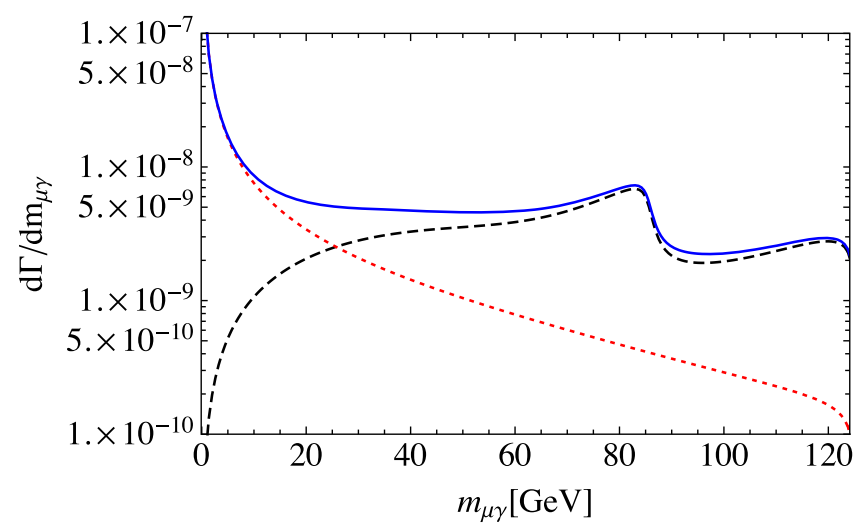

FIG. 5. Differential decay rate with respect to the invariant mass $\sqrt{t} \equiv m_{\mu \gamma}$ of the muon-photon pair. The tree-level, one-loop, and total contribution are denoted by red dotted, black dashed lines, and solid blue lines, respectively. No cuts on $s$ are introduced.

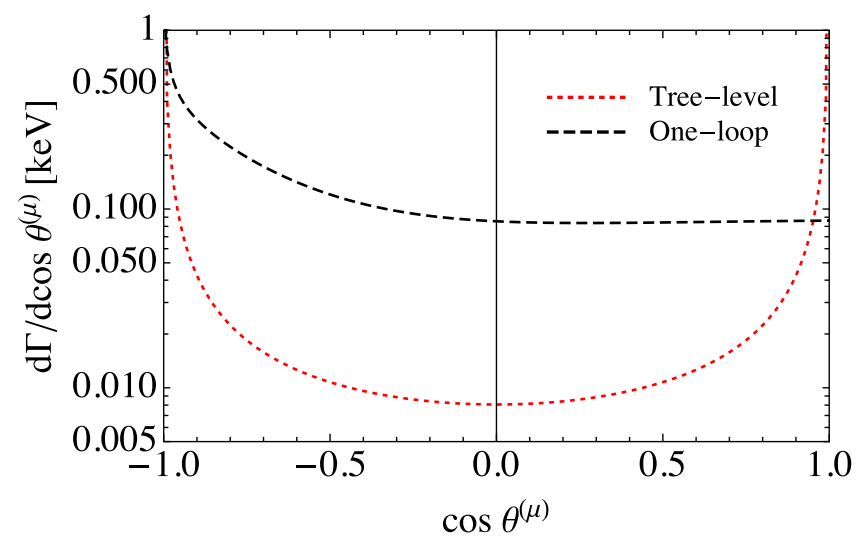

FIG. 6. Differential decay rate with respect to $\cos \theta^{(\mu)}$, where $\theta^{(\mu)}$ is the angle between the lepton and the photon in the rest frame of the Higgs boson. For the integration over $m_{\mu \mu}$ we apply the cuts $m_{\mu \mu}>0.1 m_{H}$ and $E_{\gamma, \min }=5 \mathrm{GeV}$.

With the cuts $m_{\ell \ell}>0.1 m_{H}$ and $E_{\gamma, \min }=5 \mathrm{GeV}$, applied to both the numerator and the denominator in Eq. (11), we obtain the numerical values

$$
\mathcal{A}_{\mathrm{FB}}^{(e)}=0.343, \quad \mathcal{A}_{\mathrm{FB}}^{(\mu)}=0.255 .
$$

We note that the contribution of the tree-level diagrams to the numerators of the asymmetries in Eq. (11) can be neglected relatively to the dominant one-loop contribution and the numerators for the electron and muon case are essentially identical. Thus the difference between $\mathcal{A}_{\mathrm{FB}}^{(e)}$ and $\mathcal{A}_{\mathrm{FB}}^{(\mu)}$ in Eq. (12) stems from the denominators; the nonnegligible tree-level contribution increases the full rate in the muon case. The difference between $\mathcal{A}_{\mathrm{FB}}^{(e)}$ and $\mathcal{A}_{\mathrm{FB}}^{(\mu)}$ is numerically more pronounced than the one between $\Gamma^{(e)}$ and $\Gamma^{(\mu)}$ in Eq. (9), because different cuts are used in Eqs. (9) and (12).

\section{Comparison with previous results}

The main goal of our paper is the resolution of the discrepancies between the different results in the literature. Only Abbasabadi et al. [38] and Chen, Qiao, and Zhu [39] provided an analytic result. The latter paper is the only one containing terms with the Levi-Civita tensor and the origin and cancellation of such terms is discussed above in Sec. II A. In the case of the former paper we have numerically evaluated the presented formula (taking into account the typo reported in Ref. [40]) and only find quantitative agreement in some regions, while we significantly disagree in others. In the next step we have digitalized the plots for $d \Gamma\left(H \rightarrow \ell^{+} \ell^{-} \gamma\right) / d m_{e e}$ of Refs. [40,41], which have used the same cuts on the kinematic variables. We compare the two results and ours in Fig. 7. From this plot we can see that the difference between the previous works in Refs. [40,41] is up to $30 \%$. 


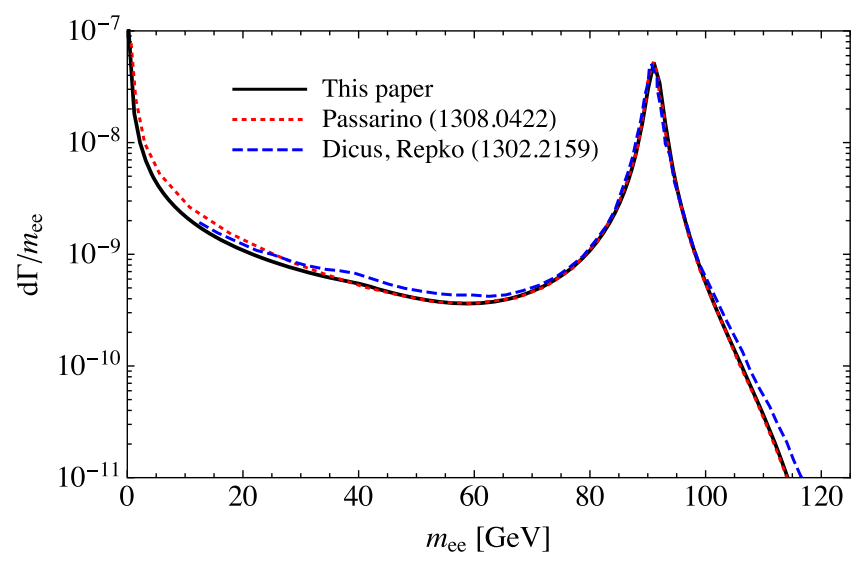

FIG. 7. Differential decay rate with respect to the invariant dilepton mass for electrons. Our result is denoted by a black solid line, while the results of Refs. [40,41] are denoted by red shortdashed and blue long-dashed lines, respectively.

Our result is close to the one of Ref. [41] for $m_{e e} \gtrsim 40 \mathrm{GeV}$ but significantly deviates for smaller values of $m_{e e}$. One may speculate that the choice of the QED fine structure constant $\alpha$, which fixes the $e^{4}$ term in the overall normalization constant $\mathcal{N}$ in Eq. (4), might account for the difference in at least the region with $m_{e e} \gtrsim 35 \mathrm{GeV}$. Shifting $\alpha^{-1}$ from the value in Eq. (8) to $\alpha^{-1}=128$ only alleviates the tension in Fig. 7 for $m_{e e} \lesssim 40 \mathrm{GeV}$ but does not fully resolve it. Furthermore, the agreement with the total decay rate becomes worse. Furthermore the shape of the distributions is different, and eventually the numerical integration over $t$ is the reason for this discrepancy. We have cross-checked our result by using different Monte Carlo generators, i.e., Vegas and the one implemented in Mathematica. Our result for the integrated rate $\Gamma^{(e)}=0.249 \mathrm{keV}$ is in reasonable agreement with the result $\Gamma^{(e)}=0.233 \mathrm{keV}$ given in Ref. [41]. We further remark that we disagree with Ref. [41] in the treelevel contribution to the integrated decay rate of $H \rightarrow \mu \bar{\mu} \gamma$ amplitude by a factor of 2 .

Reference [42] further presents results for $d \Gamma(H \rightarrow$ $\left.\ell^{+} \ell^{-} \gamma\right) / d m_{e e}$ for a different choice of cuts, namely:

$$
\Delta R_{\gamma e^{+}}>0.4, \quad \Delta R_{\gamma e^{-}}>0.4,
$$

where $\Delta R_{\gamma f}=\left(\Delta \eta^{2}+\Delta \phi^{2}\right)^{1 / 2}$ denotes the rapidityazimuthal angle separation. Digitalizing the plot in this paper as well, we compare the presented results with ours in Fig. 8. We have found that the effect of the cuts in Eq. (13) does not alter the loop-induced distribution by more than $2 \%$ in the region where we observe deviations from Ref. [42]. Therefore, we add in the same figure the digitalized result of Ref. [40] that does not employ any cut. We observe good agreement between Refs. $[40,42]$ in a region below the $Z$ peak, where we agree with these results well for $m_{\mu \mu} \gtrsim 70 \mathrm{GeV}$, while deviating otherwise. For $m_{\mu \mu}>M_{Z}$ we agree well with the result of Ref. [42].

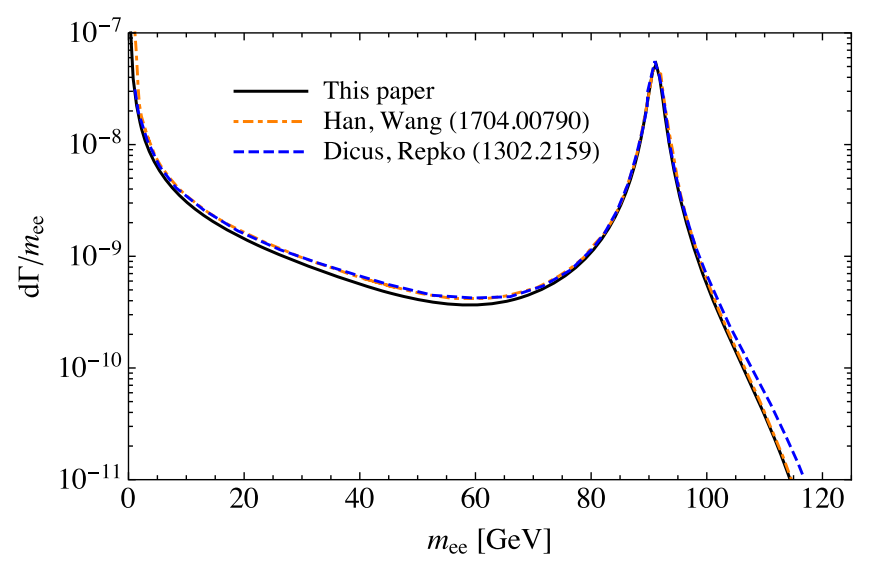

FIG. 8. Differential decay rate with respect to the invariant dilepton mass for the electron case. Our result is denoted by a black solid line, while the results of Refs. $[40,42]$ are denoted by blue dashed and orange dash-dotted lines, respectively.

\section{CONCLUSIONS}

The results in the literature for the differential decay rates $d \Gamma\left(H \rightarrow \ell^{+} \ell^{-} \gamma\right) / d m_{\ell \ell}$ with $\ell=e, \mu$ differ substantially. We have performed a new calculation of the differential decay rate $d^{2} \Gamma\left(H \rightarrow \ell^{+} \ell^{-} \gamma\right) /(d s d t)$, where $s$ is the squared invariant mass of the lepton-antilepton pair and $t$ is the corresponding quantity for the lepton-photon pair. We have performed our calculation in an $R_{\xi}$ gauge and have verified the gauge independence of the result. After presenting various differential decay distributions we have studied the forward-backward asymmetry defined in terms of the flight direction of the photon with respect to the lepton. These asymmetries, quoted in Eq. (12), are sizable.

In experimental studies one defines cuts in the laboratory reference frame rather than the Higgs rest frame and the comparison between data and SM prediction requires the use of the fully differential decay rate. For this purpose we present an analytic expression in a compact form in Appendix A and provide ancillary files for the use by experimental collaborations.

\section{ACKNOWLEDGMENTS}

We thank Vladyslav Shtabovenko for useful discussions and valuable insights into FeynCalc as well as Wayne Repko, Giampero Passarino, and Xing Wang for helpful correspondence on Refs. [38,40-42]. The research of U.N. is supported by BMBF under Grant No. 05H2018 (ErUM-FSP T09)—BELLE II: Theoretische Studien zur Flavourphysik. A. K. acknowledges the support from the doctoral school Karlsruhe School of Elementary Particle Physics and Astroparticle Physics: Science and Technology (KSETA) and the Graduate School Scholarship Program of the German Academic Exchange Service (DAAD). 


\section{APPENDIX A: EXPLICIT RESULTS}

In this section we quote the formulas for $a_{1}$ and $b_{1}$, which characterize the decay rate in Eq. (5) and are introduced in Eq. (2), in terms of the coefficient functions appearing in the Passarino-Veltman decomposition of the tensor integrals. We follow the conventions of FeynCalc [55,56].
In the equations below we use $D \equiv 4-2 \epsilon$ which appears in the coefficients of the UV-divergent loop function $B_{0}$. The products are understood to be expanded in $\epsilon$ to order $\epsilon^{0}$. One easily verifies that the $1 / \epsilon$ pole vanishes from $a_{1}$ and $b_{1}$ in the sum of the various terms involving $B_{0}$.

The function $a_{1}$ reads

$$
\begin{aligned}
a_{1}= & \frac{e^{4}}{(2 \pi)^{2}}\left[\frac{8 m_{t}^{2}\left((D-4) m_{H}^{2}-(D-2) s\right) B_{0}\left(m_{H}^{2}, m_{t}^{2}, m_{t}^{2}\right)}{3 m_{W} \sin \theta_{W}(D-2) s\left(m_{H}^{2}-s\right)^{2}}+\frac{16 m_{t}^{2} B_{0}\left(s, m_{t}^{2}, m_{t}^{2}\right)}{3 m_{W} \sin \theta_{W}(D-2)\left(m_{H}^{2}-s\right)^{2}}\right. \\
& -\frac{\left(2(D-1) m_{W}^{2}+m_{H}^{2}\right)\left((D-4) m_{H}^{2}-(D-2) s\right) B_{0}\left(m_{H}^{2}, m_{W}^{2}, m_{W}^{2}\right)}{2 m_{W} \sin \theta_{W}(D-2) s\left(m_{H}^{2}-s\right)^{2}}-\frac{\left(2(D-1) m_{W}^{2}+m_{H}^{2}\right) B_{0}\left(s, m_{W}^{2}, m_{W}^{2}\right)}{m_{W} \sin \theta_{W}(D-2)\left(m_{H}^{2}-s\right)^{2}} \\
& -\frac{2 m_{t}^{2}\left(-2 m_{H}^{2}+2 s+8 m_{t}^{2}\right) C_{0}\left(0, m_{H}^{2}, s, m_{t}^{2}, m_{t}^{2}, m_{t}^{2}\right)}{3 m_{W} \sin \theta_{W} s\left(m_{H}^{2}-s\right)}+\frac{m_{W}\left(-3 m_{H}^{2}+6 m_{W}^{2}+4 s\right) C_{0}\left(0, m_{H}^{2}, s, m_{W}^{2}, m_{W}^{2}, m_{W}^{2}\right)}{\sin \theta_{W} s\left(m_{H}^{2}-s\right)} \\
& +\frac{m_{Z} \sin \theta_{W}}{\cos ^{3} \theta_{W}}\left(D_{23}\left(0, u, 0, t, 0, m_{H}^{2}, 0, m_{Z}^{2}, 0, m_{Z}^{2}\right)+\left(D_{23} \rightarrow D_{33}\right)\right) \\
& +\frac{1}{s-m_{Z}^{2}+i m_{Z} \Gamma_{Z}}\left(\frac{\left(4 \cos \theta_{W}^{2}-1\right) m_{Z} C_{0}\left(0, m_{H}^{2}, s, m_{W}^{2}, m_{W}^{2}, m_{W}^{2}\right)}{\cos \theta_{W} \sin \theta_{W}}\right. \\
& +\frac{\left(5-8 \cos \theta_{W}^{2}\right) m_{t}^{2} C_{0}\left(0, m_{H}^{2}, s, m_{t}^{2}, m_{t}^{2}, m_{t}^{2}\right)}{6 \cos ^{2} \theta_{W} \sin \theta_{W} m_{W}}+\frac{2\left(8 \cos \theta_{W}^{2}-5\right) m_{t}^{2} C_{12}\left(0, m_{H}^{2}, s, m_{t}^{2}, m_{t}^{2}, m_{t}^{2}\right)}{3 \cos ^{2} \theta_{W} \sin \theta_{W} m_{W}} \\
& \left.\left.-\frac{\left(2 m_{W}^{2}\left(6 \cos ^{2} \theta_{W}-1\right)+\left(2 \cos ^{2} \theta_{W}-1\right) m_{H}^{2}\right) C_{12}\left(0, m_{H}^{2}, s, m_{W}^{2}, m_{W}^{2}, m_{W}^{2}\right)}{2 \cos ^{2} \theta_{W} \sin \theta_{W} m_{W}}\right)\right] .
\end{aligned}
$$

The explicit form of the function $b_{1}$ is

$$
\begin{aligned}
& b_{1}=\frac{e^{4}}{(2 \pi)^{2}}\left[\frac{8\left((D-4) m_{H}^{2}-(D-2) s\right) B_{0}\left(m_{H}^{2}, m_{t}^{2}, m_{t}^{2}\right) m_{t}^{2}}{3 m_{W} \sin \theta_{W}(D-2)\left(m_{H}^{2}-s\right)^{2} s}+\frac{16 B_{0}\left(s, m_{t}^{2}, m_{t}^{2}\right) m_{t}^{2}}{3 m_{W} \sin \theta_{W}(D-2)\left(m_{H}^{2}-s\right)^{2}}\right. \\
& -\frac{\left(m_{H}^{2}+2(D-1) m_{W}^{2}\right) B_{0}\left(s, m_{W}^{2}, m_{W}^{2}\right)}{m_{W} \sin \theta_{W}(D-2)\left(m_{H}^{2}-s\right)^{2}}-\frac{\left(m_{H}^{2}+2(D-1) m_{W}^{2}\right)\left((D-4) m_{H}^{2}-(D-2) s\right) B_{0}\left(m_{H}^{2}, m_{W}^{2}, m_{W}^{2}\right)}{2 m_{W} \sin \theta_{W}(D-2)\left(m_{H}^{2}-s\right)^{2} s} \\
& +\frac{2\left(2 m_{H}^{2}-8 m_{t}^{2}-2 s\right) C_{0}\left(0, m_{H}^{2}, s, m_{t}^{2}, m_{t}^{2}, m_{t}^{2}\right) m_{t}^{2}}{3 m_{W} \sin \theta_{W}\left(m_{H}^{2}-s\right) s}+\frac{m_{W}\left(-3 m_{H}^{2}+6 m_{W}^{2}+4 s\right) C_{0}\left(0, m_{H}^{2}, s, m_{W}^{2}, m_{W}^{2}, m_{W}^{2}\right)}{\sin \theta_{W}\left(m_{H}^{2}-s\right) s} \\
& -\frac{m_{W} D_{0}\left(m_{H}^{2}, 0,0,0, s, t, m_{W}^{2}, m_{W}^{2}, m_{W}^{2}, 0\right)}{2 \sin ^{3} \theta_{W}}-\frac{m_{W} D_{1}\left(0, t, m_{H}^{2}, s, 0,0, m_{W}^{2}, 0, m_{W}^{2}, m_{W}^{2}\right)}{2 \sin ^{3} \theta_{W}} \\
& +\frac{m_{W} D_{3}\left(0, u, m_{H}^{2}, s, 0,0, m_{W}^{2}, 0, m_{W}^{2}, m_{W}^{2}\right)}{2 \sin ^{3} \theta_{W}}-\frac{m_{W} D_{3}\left(0, t, m_{H}^{2}, s, 0,0, m_{W}^{2}, 0, m_{W}^{2}, m_{W}^{2}\right)}{2 \sin ^{3} \theta_{W}} \\
& +\frac{m_{W} D_{12}\left(0, t, m_{H}^{2}, s, 0,0, m_{W}^{2}, 0, m_{W}^{2}, m_{W}^{2}\right)}{2 \sin ^{3} \theta_{W}}+\frac{m_{Z}\left(1-2 \cos ^{2} \theta_{W}\right)^{2} D_{23}\left(0, u, 0, t, 0, m_{H}^{2}, 0, m_{Z}^{2}, 0, m_{Z}^{2}\right)}{4 \cos ^{3} \theta_{W} \sin ^{3} \theta_{W}} \\
& +\frac{m_{W} D_{23}\left(0, u, m_{H}^{2}, s, 0,0, m_{W}^{2}, 0, m_{W}^{2}, m_{W}^{2}\right)}{2 \sin ^{3} \theta_{W}}+\frac{m_{W} D_{23}\left(0, t, m_{H}^{2}, s, 0,0, m_{W}^{2}, 0, m_{W}^{2}, m_{W}^{2}\right)}{2 \sin ^{3} \theta_{W}} \\
& +\frac{m_{Z}\left(1-2 \cos ^{2} \theta_{W}\right)^{2} D_{33}\left(0, u, 0, t, 0, m_{H}^{2}, 0, m_{Z}^{2}, 0, m_{Z}^{2}\right)}{4 \cos ^{3} \theta_{W} \sin ^{3} \theta_{W}} \\
& -\frac{1}{s-m_{Z}^{2}+i m_{Z} \Gamma_{Z}}\left(\frac{\left(2 \cos ^{2} \theta_{W}-1\right)\left(4 \cos ^{2} \theta_{W}-1\right) m_{Z} C_{0}\left(0, m_{H}^{2}, s, m_{W}^{2}, m_{W}^{2}, m_{W}^{2}\right)}{2 \cos \theta_{W} \sin ^{3} \theta_{W}}\right. \\
& +\frac{\left(5-8 \cos ^{2} \theta_{W}\right)\left(2 \cos ^{2} \theta_{W}-1\right) m_{t}^{2} C_{0}\left(0, m_{H}^{2}, s, m_{t}^{2}, m_{t}^{2}, m_{t}^{2}\right)}{12 \cos ^{2} \theta_{W} \sin ^{3} \theta_{W} m_{W}}+\frac{\left(2 \cos ^{2} \theta_{W}-1\right)\left(8 \cos ^{2} \theta_{W}-5\right) m_{t}^{2} C_{12}\left(0, m_{H}^{2}, s, m_{t}^{2}, m_{t}^{2}, m_{t}^{2}\right)}{3 \cos ^{2} \theta_{W} \sin ^{3} \theta_{W} m_{W}} \\
& \left.\left.-\frac{\left(2 \cos ^{2} \theta_{W}-1\right)\left(2 m_{W}^{2}\left(6 \cos ^{2} \theta_{W}-1\right)+\left(2 \cos ^{2} \theta_{W}-1\right) m_{H}^{2}\right) C_{12}\left(0, m_{H}^{2}, s, m_{W}^{2}, m_{W}^{2}, m_{W}^{2}\right)}{4 \cos ^{2} \theta_{W} \sin ^{3} \theta_{W} m_{W}}\right)\right] .
\end{aligned}
$$




\section{APPENDIX B: USAGE OF THE ANCILLARY FILES}

In the ancillary file attached to the arXiv preprint we provide the analytical expressions for the one-loop coefficients $a_{i}, b_{i}, i=1,2$, defined in Eq. (2). The file coeff_nonreduced.m contains the results that match Eqs. (A1) and (A2), while coeff_reduced.m contains the same results in the form that is reduced to the standard basis $\left\{A_{0}, B_{0}, C_{0}, D_{0}\right\}$ of scalar one-loop functions. The coefficients are provided in both FeynCalc and Package-X notations and conventions. After importing the files, the coefficients can be called using, for example, a1PXr for the coefficient $a_{1}$ in the reduced form and Package-X notation, or b1FCnr for coefficient $b_{1}$ in the nonreduced form and FeynCalc notation. For instance, the coefficients given in the Package-X notation can be numerically evaluated using Collier [60] via the CollierLink [57] package; the detailed manual for the latter package can be found in [59].
[1] S. Chatrchyan et al. (CMS Collaboration), Phys. Lett. B 716, 30 (2012).

[2] G. Aad et al. (ATLAS Collaboration), Phys. Lett. B 716, 1 (2012).

[3] C. Englert, A. Freitas, M. M. Mühlleitner, T. Plehn, M. Rauch, M. Spira, and K. Walz, J. Phys. G 41, 113001 (2014).

[4] G. Aad et al. (ATLAS Collaboration), J. High Energy Phys. 08 (2015) 137.

[5] G. Aad et al. (ATLAS Collaboration), Phys. Rev. D 92, 012006 (2015).

[6] A. M. Sirunyan et al. (CMS Collaboration), Phys. Lett. B 791, 96 (2019).

[7] A. M. Sirunyan et al. (CMS Collaboration), Phys. Rev. D 99, 112003 (2019).

[8] S. Chatrchyan et al. (CMS Collaboration), Phys. Rev. D 89, 092007 (2014).

[9] G. Aad et al. (ATLAS Collaboration), Phys. Lett. B 738, 234 (2014).

[10] M. Aaboud et al. (ATLAS Collaboration), Phys. Lett. B 786, 114 (2018).

[11] G. Aad et al. (ATLAS Collaboration), J. High Energy Phys. 04 (2015) 117.

[12] M. Aaboud et al. (ATLAS Collaboration), Phys. Rev. D 99, 072001 (2019).

[13] A. M. Sirunyan et al. (CMS Collaboration), Phys. Lett. B 779, 283 (2018).

[14] A. M. Sirunyan et al. (CMS Collaboration), Phys. Rev. Lett. 121, 121801 (2018).

[15] M. Aaboud et al. (ATLAS Collaboration), Phys. Lett. B 786, 59 (2018).

[16] A. M. Sirunyan et al. (CMS Collaboration), J. High Energy Phys. 08 (2018) 066.

[17] M. Aaboud et al. (ATLAS Collaboration), Phys. Lett. B 784, 173 (2018).

[18] A. M. Sirunyan et al. (CMS Collaboration), J. High Energy Phys. 11 (2018) 185.

[19] M. Aaboud et al. (ATLAS Collaboration), Phys. Rev. D 98, 052005 (2018).

[20] O. Eberhardt, G. Herbert, H. Lacker, A. Lenz, A. Menzel, U. Nierste, and M. Wiebusch, Phys. Rev. Lett. 109, 241802 (2012).

[21] O. Eberhardt, U. Nierste, and M. Wiebusch, J. High Energy Phys. 07 (2013) 118.
[22] G. Belanger, B. Dumont, U. Ellwanger, J. F. Gunion, and S. Kraml, Phys. Rev. D 88, 075008 (2013).

[23] J. Baglio, O. Eberhardt, U. Nierste, and M. Wiebusch, Phys. Rev. D 90, 015008 (2014).

[24] S. Kanemura, K. Tsumura, K. Yagyu, and H. Yokoya, Phys. Rev. D 90, 075001 (2014).

[25] P. S. Bhupal Dev and A. Pilaftsis, J. High Energy Phys. 12 (2014) 024; 11 (2015) 147(E).

[26] A. Broggio, E. J. Chun, M. Passera, K. M. Patel, and S. K. Vempati, J. High Energy Phys. 11 (2014) 058.

[27] D. Chowdhury and O. Eberhardt, J. High Energy Phys. 11 (2015) 052.

[28] J. Bernon, J. F. Gunion, H. E. Haber, Y. Jiang, and S. Kraml, Phys. Rev. D 92, 075004 (2015).

[29] H. E. Haber and O. Stål, Eur. Phys. J. C 75, 491 (2015); 76, 312(E) (2016).

[30] L. Wang, F. Zhang, and X. F. Han, Phys. Rev. D 95, 115014 (2017).

[31] D. Chowdhury and O. Eberhardt, J. High Energy Phys. 05 (2018) 161.

[32] A. Keshavarzi, D. Nomura, and T. Teubner, Phys. Rev. D 97, 114025 (2018).

[33] R. Aaij et al. (LHCb Collaboration), Phys. Rev. Lett. 122, 191801 (2019).

[34] R. Aaij et al. (LHCb Collaboration), J. High Energy Phys. 08 (2017) 055.

[35] M. Algueró, B. Capdevila, A. Crivellin, S. Descotes-Genon, P. Masjuan, J. Matias, and J. Virto, Eur. Phys. J. C 79, 714 (2019).

[36] J. Aebischer, W. Altmannshofer, D. Guadagnoli, M. Reboud, P. Stangl, and D. M. Straub, arXiv:1903.10434.

[37] A. M. Sirunyan et al. (CMS Collaboration), J. High Energy Phys. 11 (2018) 152.

[38] A. Abbasabadi, D. Bowser-Chao, D. A. Dicus, and W. W. Repko, Phys. Rev. D 55, 5647 (1997).

[39] L. B. Chen, C. F. Qiao, and R. L. Zhu, Phys. Lett. B 726, 306 (2013).

[40] D. A. Dicus and W. W. Repko, Phys. Rev. D 87, 077301 (2013).

[41] G. Passarino, Phys. Lett. B 727, 424 (2013).

[42] T. Han and X. Wang, J. High Energy Phys. 10 (2017) 036.

[43] A. Denner, S. Dittmaier, M. Roth, and D. Wackeroth, Nucl. Phys. B560, 33 (1999). 
[44] A. Denner and S. Dittmaier, Nucl. Phys. B, Proc. Suppl. 160, 22 (2006).

[45] M. Aaboud et al. (ATLAS Collaboration), J. High Energy Phys. 10 (2017) 112.

[46] A. M. Sirunyan et al. (CMS Collaboration), J. High Energy Phys. 09 (2018) 148.

[47] L. M. Brown and R. P. Feynman, Phys. Rev. 85, 231 (1952).

[48] G. Passarino and M. J. G. Veltman, Nucl. Phys. B160, 151 (1979).

[49] G. 't Hooft and M. J. G. Veltman, Nucl. Phys. B153, 365 (1979).

[50] A. Denner, U. Nierste, and R. Scharf, Nucl. Phys. B367, 637 (1991).

[51] See Supplemental Material at http://link.aps.org/ supplemental/10.1103/PhysRevD.101.073003, for the analytic results for the loop functions $a_{1,2}$ and $b_{1,2}$.

[52] Y. Sun, H. R. Chang, and D. N. Gao, J. High Energy Phys. 05 (2013) 061.

[53] A. Abbasabadi, D. Bowser-Chao, D. A. Dicus, and W. W. Repko, Phys. Rev. D 52, 3919 (1995).

[54] T. Hahn, Comput. Phys. Commun. 140, 418 (2001).

[55] V. Shtabovenko, R. Mertig, and F. Orellana, Comput. Phys. Commun. 207, 432 (2016).

[56] R. Mertig, M. Bohm, and A. Denner, Comput. Phys. Commun. 64, 345 (1991).

[57] H. H. Patel, Comput. Phys. Commun. 197, 276 (2015).
[58] V. Shtabovenko, Comput. Phys. Commun. 218, 48 (2017).

[59] H. Patel, https://packagex.hepforge.org/Documentation/ HTML/X/tutorial/LinkingToCOLLIER.html.

[60] A. Denner, S. Dittmaier, and L. Hofer, Comput. Phys. Commun. 212, 220 (2017).

[61] A. Denner and S. Dittmaier, Nucl. Phys. B734, 62 (2006).

[62] A. Denner and S. Dittmaier, Nucl. Phys. B844, 199 (2011).

[63] T. Hahn and M. Perez-Victoria, Comput. Phys. Commun. 118, 153 (1999).

[64] T. Hahn, http://www.feynarts.de/looptools/LT215Guide.pdf.

[65] G. P. Lepage, J. Comput. Phys. 27, 192 (1978).

[66] T. Hahn, Comput. Phys. Commun. 168, 78 (2005).

[67] Mathematica, Wolfram Research Inc., Champaign, IL, 2019.

[68] D. de Florian et al. (LHC Higgs Cross Section Working Group), arXiv:1610.07922, https://doi.org/10.23731/CYRM -2017-002.

Correction: The previously published Figs. 4-8 contained errors and have been fixed. The following elements contained mistakes and have been fixed: $B(H)$ and $\mathcal{A}_{\mathrm{FB}}$ values in the abstract, Eqs. (7), (9), (10), (12), (A1), and (A2), and the sentence after Eq. (7) has been deleted. Related elements have been modified in the Supplemental Material. 